\title{
Do neutrino oscillations allow an extra phenomenological parameter?
}

\author{
I.S. Tsukerman \\ State Research Center \\ "Institute for Theoretical and Experimental Physics" \\ Moscow, 117218, Russia \\ E-mail:zuckerma@heron.itep.ru \\ Submitted to JETP Letters
}

\begin{abstract}
The quantity $\xi$ introduced recently in the phenomenological description of neutrino oscillations is in fact not a free parameter, but a fixed number.
\end{abstract}

The literature on phenomenology of neutrino oscillations is vast (see, e.g., [1] - [6] and references therein). In a recent paper [7] Giunti and Kim in the case of two-flavour mixing have introduced a new phenomenological parameter $\xi$. According to [7], $\xi=0$ corresponds to the so-called equal momentum assumption [1], 国, while $\xi=1$ corresponds to equal energy assumption [5, 6]. Authors of [7] emphasize that $\xi$ disappears from final expressions for the neutrino oscillation probability.

The aim of this note is to indicate that parameter $\xi$ is fixed by energy-momentum conservation in the process which is responsible for neutrino emission, as explicitly assumed in ref. [7].

Following ref. [7] we will consider the decay $\pi \rightarrow \mu \nu$ in the framework of two-flavour toy model. The parameter $\xi$ is defined in [7] for the pion rest-frame by considering the auxiliary case of absolutely massless neutrinos and denoting the energy of such neutrinos as $E$,

$$
\xi={ }^{1} / 2\left(1+m_{\mu}^{2} / m_{\pi}^{2}\right),
$$

where $m_{\mu}$ and $m_{\pi}$ are the masses of the muon and the pion. Then for massive (but light!) neutrinos they get:

$$
\begin{gathered}
E_{1,2}=E+(1-\xi) m_{1,2}^{2} / 2 E \\
p_{1,2}=E-\xi m_{1,2}^{2} / 2 E .
\end{gathered}
$$

Here $E_{1,2}, p_{1,2}$ and $m_{1,2}$ are the energies, momenta and masses of the neutrinos, respectively. Wherefrom the above statement about $\xi=0,1$ follows:

$$
E_{1}=E_{2} \text { for } \xi=1 \text { and } p_{1}=p_{2} \text { for } \xi=0 .
$$

Thus, the equal energy and equal momentum assumptions in the form $\Delta E \equiv E_{1}-E_{2}=0$ and $\Delta p \equiv p_{1}-p_{2}=0$, respectively, are treated by authors of ref. [7] as particular cases of the general kinematical relations (2) and (3):

$$
\Delta E=(1-\xi) \Delta m^{2} / 2 E=0 \text { for } \xi=1,
$$




$$
\Delta p=\xi \Delta m^{2} / 2 E=0 \text { for } \xi=0 .
$$

Unfortunately, both the treatment and the relations (何) - (6) are erroneous.

On one hand, the quantity $\xi$ is not a free parameter for a certain decay process. Indeed, it follows from (四) that $\xi$ has a fixed value $(\simeq 0.8)$ for the decay under consideration. On the other hand, it is evident from definitions of $E$ and $\xi$ that

$$
E=m_{\pi}(1-\xi) .
$$

The parameter $\xi$ determines sharing of the decay energy. As seen from (7), the values $\xi=0$ and $\xi=1$ are senseless ones because they refer accordingly to the limiting cases of $E_{\mu}=0$ and $E=0$. Therefore one cannot assume that $\xi$ can be equal to 1 or 0 . Instead of that, the solution of the equalities (5) and (6) is the vanishing $\Delta m^{2}$, that is absence of the oscillations.

\section{Acknowledgements}

The author is grateful to L.B. Okun for his friendly support. This work was supported by RFBR grant 00-15-96562.

\section{References}

[1] B.M. Pontecorvo, ZhETF 53, 1717 (1967).

[2] V.N. Gribov, B.M. Pontecorvo, Phys. Lett. 28B, 493 (1969).

[3] S. Bilenky, B. Pontecorvo, Phys. Lett. 61B, 248 (1976); Lett. Nuovo Cim. 17, 569 (1976); Comments Nucl. Part. Phys. 7, 149 (1977).

[4] L.B. Okun, Surveys in High Energy Phys. 15, 75 (2000).

[5] H. Lipkin, hep-ph/9901399.

[6] L. Stodolsky, Phys. Rev. D58, 036006 (1998); hep-ph/9802387.

[7] C. Giunti, C.W. Kim, hep-ph/0011074. 